\title{
Improved Wide-Angle, Fisheye and Omnidirectional Camera Calibration
}

\author{
Steffen Urban, Jens Leitloff, Stefan Hinz \\ Karlsruhe Institute of Technology (KIT), Institute of Photogrammetry and Remote Sensing, \\ Englerstr. 7, 76131 Karlsruhe
}

\section{Abstract}

In this paper an improved method for calibrating wide-angle, fisheye and omnidirectional imaging systems is presented. We extend the calibration procedure proposed by Scaramuzza et al. by replacing the residual function and joint refinement of all parameters. In doing so, we achieve a more stable, robust and accurate calibration (up to factor 7) and can reduce the number of necessary calibration steps from five to three. After introducing the camera model and highlighting the differences from the current calibration procedure, we perform a comprehensive performance evaluation using several data sets and show the impact of the proposed calibration procedure on the calibration results.

Keywords: Camera, Calibration, Accuracy, Precision, Geometric, Robotics, Bundle, Fisheye, Omnidirectional

\section{INTRODUCTION}

In this paper we extend an existing method to calibrate wide-angle, fisheye and omnidirectional cameras based on the generalized camera model introduced in 11. In the following, we propose contributions that not only enhance the methods implemented in [2], but also the underlying calibration procedure. Our main contribution will be the reformulation of the two step non-linear optimization to

\footnotetext{
${ }^{*}$ Corresponding author

Email address: steffen.urban@kit.edu (Steffen Urban, Jens Leitloff, Stefan Hinz)

$U R L$ : www.ipf.kit.edu (Steffen Urban, Jens Leitloff, Stefan Hinz)
} 
jointly refine all calibration parameters. We then perform various calibrations on data sets provided by the authors [2] and own data sets. Afterwards, we elaborately analyze the results and detail the significant gain in performance and accuracy. In addition, we calibrated all cameras with another calibration method proposed by [3] which is also available as a Matlab toolbox [4. The Matlab implementation of all proposed contributions as well as test data sets and evaluation scripts will be made available onlin $\varrho^{1}$

\subsection{Related Work}

Wide-angle, omnidirectional and fisheye cameras are nowadays popular in many fields of computer vision, robotics and photogrammetric tasks such as navigation, localization, tracking, mapping and so on. As soon as metric information needs to be extracted, camera calibration is the first necessary step to determine the relation between a $3 \mathrm{D}$ ray and its corresponding mapping on the image plane.

In general, camera calibration consists of two steps. The first involves the choice of an appropriate model that describes the behavior of the imaging system. In recent years various such models for dioptric (fisheye) and catadioptric (omnidirectional) cameras have been proposed [1, 5, 3, 6, 7, 8, In [9] a comprehensive overview is given. The second step is the estimation of all parameters that a certain model incorporates, i.e. interior and exterior orientation of the system as well as distortion parameters that model the differences from an ideal imaging process. Several methods for calibration have been proposed, that can be classified into three categories [10] starting with the most general one.

The first is auto- or self-calibration, where epipolar-constraints and point correspondences between multiple views are used [6, 11, 12]. In [11] the autocalibration for fisheye and catadioptric systems is based on the robust establishment of the epipolar geometry from few correspondences in two images by solving a polynomial eigenvalue problem. Using this model, Ransac is employed

\footnotetext{
${ }^{1}$ http://www.ipf.kit.edu/code.php
} 
to remove outliers and no prior knowledge about the scene is necessary. Subsequently, bundle block adjustment can be employed, to estimate all camera parameters and reconstruct the scene except for a similarity transformation.

The second is space resection of one image from a non-planar object with known 3D world coordinates [13, 14]. [13] investigate the validity of geometric projections for fisheye lenses. The camera is calibrated using one image of a room prepared with 3D-passpoints, that cover the field of view of the fisheye lens. To enhance the model quality and compensate for real world deviations from the geometric model, radial as well as tangential distortion parameters are subsequently added to the projection model. A camera calibration approach for catadioptric cameras using space resection is proposed in [14. Lifted coordinates are used to linearily project scene points to the image plane. After the projection matrix is estimated from scene points distributed over a 3D-calibration object a final non-linear refinement is applied.

The third calibration method involves the observation of a planar object (e.g. checkerboard) with known 3D object coordinates [1, 3, 10, where at least two images of the planar object are necessary [10]. The calibration procedure of 3] involves the manual selection of mirror border and center, respectively. For fisheye lenses the image center is used. The generalized focal length, which is part of the exact projection model, is estimated from at least three points on a line. To automatically extract all checkerboard corners, a planar homography is estimated from four manually selected points. Then the remaining checkerboard points are projected to their estimated location and a local subpixel corner search is performed. The initially estimated homography is based on start values such as the generalized focal length and the center of distortion and thus already imposes model assumptions. If the initial assumptions are highly biased the subsequent procedure can hardly recover. The projection model of [3] include tangential distortion parameters, whereas [1] models only radial symmetric effects. [1 initially extracts all checkerboard corners using the algorithm of [15]. The calibration procedure is based on multiple observations of 
a planar calibration object [10], is fully automatic and does not require further user interaction or prior knowledge. A potential disadvantage are wrong point extractions, that can bias the calibration result. Also the two-step refinement of interior and exterior orientation is critical, as all parameters are strongly correlated. Our proposed approach tries to avoid both possible disadvantages, but does not change the underlying projection model.

\subsection{Contributions}

In this paper we extend and improve the widespread calibration procedure of [1] and its online available implementation [2] as this does not provide an optimal solution. The camera model proposed in [1] is general and does not require prior knowledge about the imaging function. The calibration procedure is almost fully automatic, online available [2] and is conducted in five consecutive steps.

We analyzed the two step refinement of interior and exterior orientation and replaced the employed residual function in order to be able to jointly refine all parameters. Thereby we obtain better convergence behaviours of the non-linear least squares optimization and it shows that the original five step calibration procedure can be conducted in three steps. In addition, we change the original implementation to a consistent use of subpixel accurate point measurements. All changes are available online and can be used as an add-on to the original toolbox at hand.

Previous work for improving [1] was conducted by [16]. They extended the camera model by replacing the affine transformation between image and sensor plane coordinates with a perspective transformation and also proposed a new method to estimate the center of distortion. They indicate that a bundle adjustment was performed in the end without providing details. Then they conducted calibrations on several data sets and compared the results to the original implementation. They observed slight improvements of the mean squared reprojection error (MSE in pixel) but did not discuss why the standard deviation of the reprojection error was partly larger than the MSE and even larger 
than the standard deviation of the original implementation. Further, only the reprojection error was investigated to assess the calibration quality. This paper additionally examines the quality of the estimated calibration parameters.

In the remainder of this paper, we first recapitulate the camera model as well as the calibration procedure as proposed in [1]. Then, we detail our contributions to the existing approach and perform a comprehensive evaluation using data sets provided by the authors [2] as well as own data sets and show that we achieve a significant accuracy increase. The improved results are subsequently compared to a different calibration method proposed by 3 .

\section{Camera Model and Calibration}

In the following and for the sake of the self-containedness of this paper, we include a condensed compilation of the generalized camera model of [1] and the five consecutive steps that it takes to estimate all model parameters. For a detailed description and insight we refer to [1, [5]. We use a similar notation as [1] to facilitate the comparison, i.e. vectors and matrices are typed in boldface, e.g. $\mathbf{R}, \mathbf{m}$, thereby vectors are lowercase and matrices are uppercase. In addition parameters to be estimated are indicated by a hat, e.g. $\hat{\mathbf{R}}, \hat{a}$.

Subsequently, we detail the procedure that is employed to estimate all calibration related parameters. In Section 3 we propose our improvements to the given procedure and their implementation.

\subsection{Generalized Camera Model}

Let $\mathbf{m}^{\prime}=\left[u^{\prime}, v^{\prime}\right]^{T}$ be a point on the image plane, i.e. the coordinates are measured from the upper left corner of the image. Its corresponding point on the sensor plane, i.e. measured from the image center, is denoted by $\mathbf{m}=[u, v]^{T}$. Those two points, are related by an affine transformation $\mathbf{m}^{\prime}=\mathbf{A m}+\mathbf{O}_{c}$ :

$$
\left[\begin{array}{l}
u^{\prime} \\
v^{\prime}
\end{array}\right]=\left[\begin{array}{ll}
c & d \\
e & 1
\end{array}\right]\left[\begin{array}{l}
u \\
v
\end{array}\right]+\left[\begin{array}{l}
o_{u} \\
o_{v}
\end{array}\right]
$$


The transformation matrix $\mathbf{A}$ accounts for small misalignments between sensor and lens axes and the digitization process. The translation $\mathbf{O}_{c}=\left[o_{u}, o_{v}\right]^{T}$ relates all coordinates to the center of distortion. Now let $\mathbf{X}_{c}=\left[X_{c}, Y_{c}, Z_{c}\right]^{T}$ be a scene point in the camera coordinate system. Then, the following forward mapping from a $2 \mathrm{D}$ image point to its corresponding $3 \mathrm{D}$ ray is described through the imaging function $g$.

$$
\lambda g(\mathbf{m})=\lambda(u, v, f(u, v))^{T}=\lambda(u, v, f(\rho))^{T}=\mathbf{X}_{c}
$$

with $\lambda>0$ and $\rho=\sqrt{u^{2}+v^{2}}$ being the radial euclidean distance from the image center. Thus, the mapping $g$ is assumed to be rotationally symmetric. Function $f$ usually depends on the mirror or lens of the system and models the projection. In literature and practical applications various projection models can be found (see Section 1.1). Instead of modelling the projection with a specific mapping function, [1] chose to approximate it with a Taylor expansion making it applicable for various lenses without prior knowledge:

$$
f(\rho)=a_{0}+a_{2} \rho^{2}+\ldots+a_{n} \rho^{n}
$$

In the next section the calibration procedure is detailed which is aimed at an accurate estimation of the mapping function $g$.

\subsection{Camera Calibration}

For this particular camera model, the goal of calibration is to estimate the parameters of the imaging function $g$, i.e. the $N-1$ coefficients $a_{0}, a_{2}, \ldots, a_{N}$ that describe the shape of the projection function, as well as the parameters of the affine transformation $\mathbf{A}$ and the center of distortion $\mathbf{O}_{c}$. During the first calibration steps $\mathbf{A}$ is set to be an identity matrix $\mathbf{I}_{2,2}$, thus the eccentricity of the mirror boundary is set to 0 . The inital estimate of the center of distortion $\mathbf{O}_{c}$ is always set to the image center. During camera calibration, a planar calibration pattern $\left(Z_{i}=0\right)$ is observed $j=1, . ., K$ times from an unknown position $\hat{\mathbf{P}}_{j}=\left[\hat{\mathbf{r}}_{1}, \hat{\mathbf{r}}_{2}, \hat{\mathbf{r}}_{3}, \hat{\mathbf{t}}\right]_{j}$. The $i=1, . ., L$ object coordinates are denoted by 
$\mathbf{X}_{i}=\left[X_{i}, Y_{i}, 0,1\right]^{T}$. The corresponding image points $\mathbf{m}_{i j}=\left[u_{i j}^{\prime}-o_{u}, v_{i j}^{\prime}-o_{v}\right]^{T}$ are extracted in each image. This leads to the following relation:

$$
\lambda_{i j} g(\mathbf{m})_{i j}=\lambda_{i j}\left[\begin{array}{c}
u \\
v \\
\hat{a}_{0}, . ., \hat{a}_{N} \rho^{N}
\end{array}\right]_{i j} \hat{=} \hat{\mathbf{P}}_{j} \mathbf{X}_{i}=\left[\begin{array}{lll}
\hat{\mathbf{r}}_{1} & \hat{\mathbf{r}}_{2} & \hat{\mathbf{t}}
\end{array}\right]_{j}\left[\begin{array}{c}
X \\
Y \\
1
\end{array}\right]_{i}
$$

Observe that the third column vector $\hat{\mathbf{r}}_{3}$ of $\mathbf{P}$ is omitted because $Z_{i}=0$.

\subsubsection{Linear Estimation of the Exterior and Iinterior Orientation}

In order to solve Eq. 4 for all interior orientation parameters, the $K$ exterior orientations are estimated in advance. First the scale factor $\lambda$ is eliminated for each equation by vectorially multiplying both sides of Eq. 4 with $g(\mathbf{m})_{i j}$ yielding:

$$
\left[\begin{array}{c}
v_{i}\left(\hat{r}_{31} X_{i}+\hat{r}_{32} Y_{i}+\hat{t}_{3}\right)-f\left(\rho_{i}\right)\left(\hat{r}_{21} X_{i}+\hat{r}_{22} Y_{i}+\hat{t}_{2}\right) \\
f\left(\rho_{i}\right)\left(\hat{r}_{11} X_{i}+\hat{r}_{12} Y_{i}+\hat{t}_{1}\right)-u_{i}\left(\hat{r}_{31} X_{i}+\hat{r}_{32} Y_{i}+\hat{t}_{3}\right) \\
u_{i}\left(\hat{r}_{21} X_{i}+\hat{r}_{22} Y_{i}+\hat{t}_{2}\right)-v_{i}\left(\hat{r}_{11} X_{i}+\hat{r}_{12} Y_{i}+\hat{t}_{1}\right)
\end{array}\right]=0
$$

Then, and since $X_{i}, Y_{i}, u_{i}, v_{i}$ are the known observations, the third equation which is linear in all unknowns and does not depend on $f$, can be used to get an initial estimate of the parameters of the exterior orientation. Subsequently, latter are substituted into Eq. 5 and the resulting system is solved for the interior orientation parameters. At this point a linear estimate of all involved parameters, i.e. $\hat{\mathbf{U}}=\left[\hat{\mathbf{R}}, \hat{\mathbf{T}}, \hat{a}_{0}, \hat{a}_{2}, \ldots, \hat{a}_{N}\right]^{T}$ is found.

\subsubsection{Center of Distortion}

Until now the linear calibration procedure is carried out with the assumptions that the image center is identical to the center of distortion. To find a better estimate of the center, [5] carry out an iterative search procedure, prior to performing the non-linear refinement of all parameters. First a regular grid is placed over the image around a given $\mathbf{O}_{c}$, where each grid point corresponds to a potential $\mathbf{O}_{c}$. Then a the linear calibration procedure is conducted for each grid point and the Sum of Squared Reprojection Errors (SSRE) is calculated. 
The grid point with the minimum SSRE is taken as the potential $\mathbf{O}_{c}$ and the process is repeated until the change between two potential $\mathbf{O}_{c}$ is less than 0.5 pixels $([5])$.

\subsubsection{Linear Refinement of Exterior and Interior Orientation}

Finally the linear estimation of interior and exterior orientation is conducted once more with the updated center point $\mathbf{O}_{c}$, yielding the initial start values for the two-step non-linear refinement with all parameters.

\subsubsection{Two-Step Non-linear Refinement}

The last step in the calibration procedure of [5] is a non-linear refinement involving all calibration parameters, i.e. the exterior orientation of each camera w.r.t the calibration pattern as well as the interior orientation parameters. Instead of jointly optimizing all parameters, [5] split the estimation in two steps, i.e. interior and exterior orientation are estimated separately. They state that the convergence is not affected but the runtime is decreased. The following residual is calculated for each point correspondence:

$$
r_{s}\left(\mathbf{m}^{\prime}, \hat{\mathbf{m}}^{\prime}\right)=\sqrt{\left(u^{\prime}-\hat{u}^{\prime}\right)^{2}+\left(v^{\prime}-\hat{v}^{\prime}\right)^{2}}
$$

and the non-linear least squares problem is solved:

\section{Exterior orientation}

In a first step the exterior orientation of all camera poses is optimized. The interior orientation parameters are fixed to the values obtained from the last linear estimation, thus:

$$
\min _{\hat{\mathbf{P}}}\left\|r_{s}\right\|^{2}=\min _{\hat{\mathbf{P}}} \sum_{j=1}^{K} \sum_{i=1}^{L} r_{s}\left(\mathbf{m}_{i j}^{\prime}, \hat{\mathbf{m}}_{i j}^{\prime}\left(\hat{\mathbf{P}}, \mathbf{a}, \mathbf{A}, \mathbf{O}_{c}, \mathbf{X}\right)\right)^{2}
$$

where $\mathbf{a}=\left[a_{0}, a_{2}, \ldots, a_{N}\right]$ are the polynomial coefficients. The affine matrix

$\mathbf{A}$ is initialized with the identity matrix for the first iteration.

2. Interior orientation

Now the exterior orientation is fixed to the values from the previous step 
and the interior orientation is refined, thus

$$
\min _{\hat{\mathbf{a}}, \hat{\mathbf{A}}, \hat{\mathbf{O}}_{c}}\left\|r_{s}\right\|^{2}=\min _{\hat{\mathbf{a}}, \hat{\mathbf{A}}, \hat{\mathbf{O}}_{c}} \sum_{j=1}^{K} \sum_{i=1}^{L} r_{s}\left(\mathbf{m}_{i j}^{\prime}, \hat{\mathbf{m}}_{i j}^{\prime}\left(\mathbf{P}, \hat{\mathbf{a}}, \hat{\mathbf{A}}, \hat{\mathbf{O}}_{c}, \mathbf{X}\right)\right)^{2}
$$

Observe also, that the affine matrix $\mathbf{A}$ is now estimated for the first time.

\section{Proposed Camera Calibration}

\subsection{Analysis of the Two-Step Non-Linear Refinement}

We conducted various test calibrations with different data sets and iteratively repeated the two step refinement proposed in [5], but could not reproduce a converging solution. After each iteration, we calculate the RMS (root mean square):

$$
R M S=\sqrt{\frac{\sum_{i=1}^{n}\left\|\left(\mathbf{m}_{i}^{\prime}-\hat{\mathbf{m}}_{i}^{\prime}\right)\right\|^{2}}{2 n}}
$$

where $n$ is the total number of point correspondences over all images.

Figure 1]depicts the effect of alternately refining exterior and interior orientation for the 'Fisheye1' data set (see Section 4), i.e. each iteration represents the result of one two-step refinement as detailed in the previous chapter. The result of each iteration is used for the next iteration. This example shows, that the iterative two-step procedure would not even stop after the 17-th iteration as the error still decreases. Moreover the RMS after the 17-th iteration is still above our, in the following presented method (compare to Table 1).

As one set of the parameters (Eq. 7. A, a, $\mathbf{O}_{c}$ ) is always fixed while the other set of parameters (Eq. 7. $\mathbf{P}$ ) is optimized and the RMS value is still decreasing, it is very likely that these two sets of parameters are correlated, i.e. it is unlikely that the algorithm will converge to a correct minimum by alternating Eq. 7] and Eq. 8. We also observed, that the two-step refinement yielded slightly different results (e.g. in the order of pixels for the principal point) for slightly different initial distortion centers. This indicates, that a global minimum is not attained.

Considering again Eq. 7 and Eq. 8 and the respective residual function Eq. 6. we can observe a potential disadvantage of the proposed error function. L 


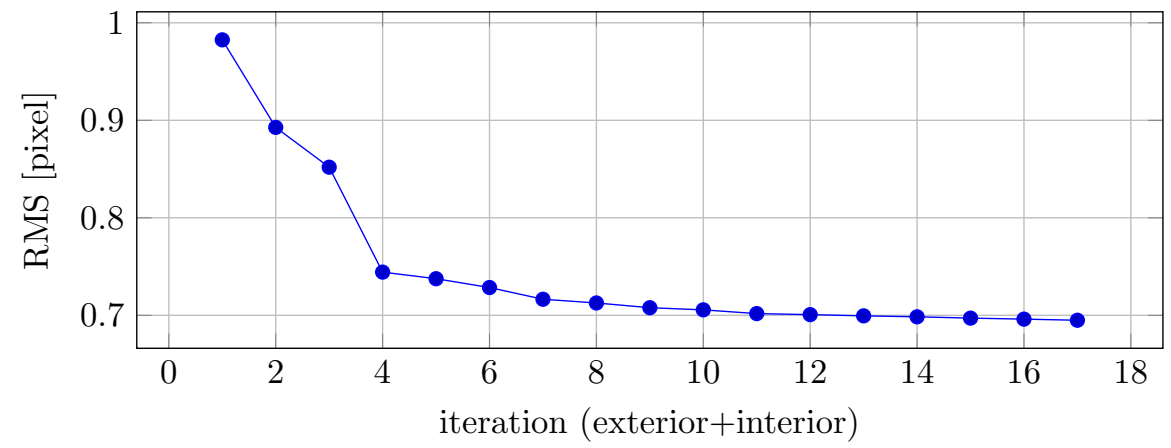

Figure 1: The RMS (root mean square) for iteratively and independently refining the exterior and interior orientation parameters as proposed in [5]. Note that the iterations refer to iterating the refinement procedure and not the iterations within each refinement. Here the 'Fisheye1' data set from Section 4 was used.

points appear in $\mathrm{K}$ images and therefore should yield $n=2 \cdot L \cdot K$ observations. Instead, Eq. 6 results in $n=L \cdot K$ observation equations. Each point actually accounts for two observations and Eq. 6incorporates both observations into one observation equation. The following section details all proposed contributions from Section 1.2 First, the implementation related contribution is described. Afterwards we detail the two contributions to the calibration methodology.

\subsection{Subpixel Corner}

The first contribution has minor impact on the methodology and is only implementation related but is not less important. If one chooses to use automatic checkerboard extraction, the toolbox [2] uses the algorithm of [15] to extract the checkerboard corners. This implementation however provides only pixel measurements up to half a pixel accuracy. We therefore changed the related source files of [2] to consistently use the corner finding method of [17] (which is already part of the toolbox) to refine all previously detected corners to subpixel accuracy. As the subpixel estimation depends on a support window size to refine the corners, it is adapted to the image size. Using a window size of $1 / 100$ of the image size proved to be a reasonable choice. 


\subsection{Non-linear Refinement}

Instead of using the original two-step optimization method of [5], we propose a method that jointly refines all calibration parameters. Using the image center, together with the linear estimated parameters obtained in Section 2.2 as initial values is sufficient for the non-linear least squares minimization to converge quickly.

The first step is to reformulate Eq. 6 and introduce a different residual function that lets us fully exploit the information at each point:

$$
r_{n e w}\left(\mathbf{m}^{\prime}, \hat{\mathbf{m}}^{\prime}, k\right)=\mathbf{m}_{k}^{\prime}-\hat{\mathbf{m}}_{k}^{\prime}
$$

where $k=1,2$ is either the $u^{\prime}$ or the $v^{\prime}$ component of the coordinate pair $\mathbf{m}^{\prime}$. Now we can substitute Eq. 10 to Eq. 7 or Eq. 8 respectively which now yields $n=2 \cdot L \cdot K$ observation equations:

$$
\min _{\hat{\mathbf{U}}}\left\|r_{n e w}\right\|^{2}=\min _{\hat{\mathbf{U}}} \sum_{j=1}^{K} \sum_{i=1}^{L} \sum_{k=1}^{2} r_{n e w}\left(\mathbf{m}_{i j}^{\prime}, \hat{\mathbf{m}}_{i j}^{\prime}(\hat{\mathbf{U}}, \mathbf{X}), k\right)^{2}
$$

with $\hat{\mathbf{U}}=\left[\hat{\mathbf{P}}, \hat{\mathbf{a}}, \hat{\mathbf{A}}, \hat{\mathbf{O}}_{c}\right]$ being the parameter vector. Following the original implementation, we minimize the non-linear least squares problem Eq. 11 using Matlab's lsqnonlin function.

At first glance, the expanded observation equations Eq. 7 and Eq. 11 should be equal. The major difference lies in the residual function and the resulting normal equations. As emphasized in Section 3.1, the original residual frunction incorporates both observations of one image point into one observation equation. Hence the finite differencing step of lsqnonlin leads to different derivations at the point of the respective unknowns. In addition, the original residual function Eq. 6] is not differentiable at the point of zero residual.

Performing several calibrations with different data sets (see Section 4 has shown, that the search for the center of distortion can be completely omitted, since the global minimum of all parameters is always found. We hereby reduce the number of necessary calibration steps to three, i.e. linear estimation of exterior and interior orientation and non-linear refinement of all parameters. 
Furthermore, the total runtime of the calibration procedure decreases as well (see Section 4.3), although the runtime of the proposed one step refinement is slightly higher than the two step refinement. Besides, latter is only true if the two step refinement is carried out only once. To obtain an adequate calibration with [5] the two step procedure has to be iterated multiple times.

\subsection{Robust Non-linear Refinement}

Usually it is not necessary to apply a robust optimization method to camera calibration. But by analyzing the extracted points from [15] we found that wrong corner points are extracted from time to time. Figure 2 depicts an example. Instead of leaving the removal of such points up to the user, we can downweight their influence during iterative application of the non-linear optimization and treat them as outliers. We do that by applying a robust M-estimator to our proposed method and performing iterative reweighted least squares:

$$
\min _{\hat{\mathbf{U}}}\left\|r_{n e w}\right\|^{2}=\min _{\hat{\mathbf{U}}} \sum_{j=1}^{K} \sum_{i=1}^{L} \sum_{k=1}^{2} \omega_{c}\left(r_{n e w}{ }^{h-1}\right) r_{n e w}\left(\mathbf{m}_{i j}^{\prime}, \hat{\mathbf{m}}_{i j}^{\prime}(\hat{\mathbf{U}}, \mathbf{X}), k\right)^{2}
$$

where the superscript $h$ indicates the $h$-th iteration. For the weight function $\omega$ we chose Huber's function:

$$
\omega_{c}(r)= \begin{cases}1, & |r| \leq c \\ c /|r|, & |r|>c\end{cases}
$$

with $c=1$ is the tuning constant. For residuals below 1 pixel the function behaves like normal least squares. All pixels with residuals larger than 1 pixel get downweighted.

\section{Performance Evaluation}

In the following, we evaluate the impact of the contributions on the calibration results. In order to provide a comprehensive evaluation, images provided by [2] containing omnidirectional and fisheye cameras as well as three own data sets of fisheye and wide-angle cameras are used. We hereby covered different camera 


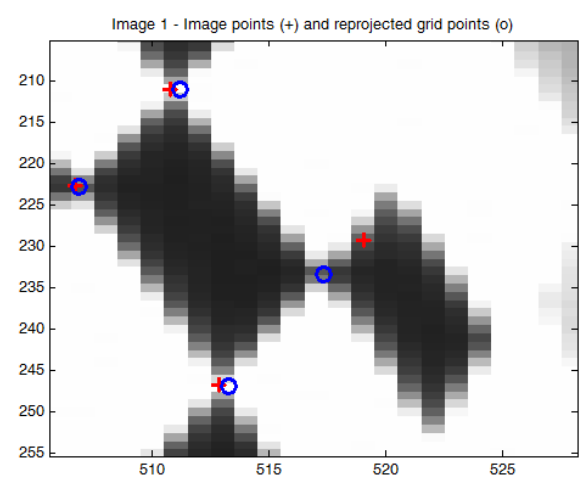

(a) bundle adjustment

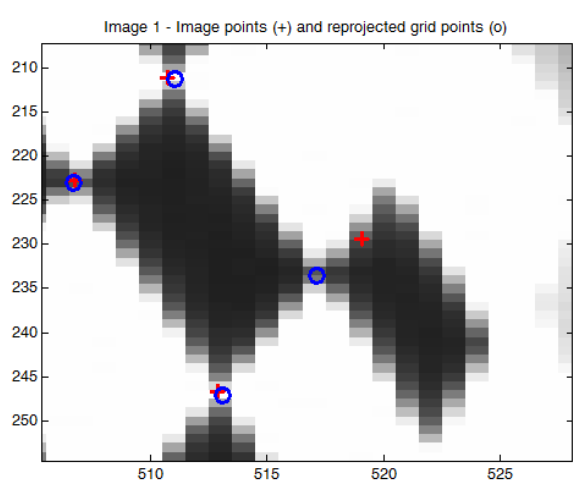

(b) robust bundle adjustment

Figure 2: Influence of wrong point extraction. The red crosses denote the extracted checkerboard points. The blue circles are the reprojected points. (a) All reprojected points are slightly biased towards the wrong checkerboard point. (b) By downweighting that point the reprojected points almost coincide with all extracted points and the bias is reduced.

models from small low-cost fisheye lenses to larger consumer cameras like the GoPro as well as omnidirectional cameras. To give the reader the possibility to reproduce all results, the data sets as well as the evaluation scripts will be made available online.

To facilitate the comparison to all real world data sets we name them after their corresponding filename. Our own data sets are called ['Fisheye1', 'Fisheye2', 'GoPro']. Data set Fisheye1 was captured with lenses from Lensation (BF2M15520) having a focal length of $1.55 \mathrm{~mm}$ and a field of view of approx. $185^{\circ}$ mounted on GiGE Vision cameras. Data set Fisheye2 was also captured with lenses from Lensation (BF2M12520) with a focal length of $1.25 \mathrm{~mm}$ and a field of view of approx. $185^{\circ}$ mounted on a USB-platform from VRmagic. The data sets provided by [2] are called ['MiniOmni', 'VMRImage', 'Ladybug', 'KaidanOmni'] and consist of three omnidirectional cameras and one wide-angle camera. Appart from the RMS (root mean square) value, we analyze the covariance matrices after the non-linear least squares estimation and extract standard deviations for the estimated parameters. 


\subsection{Influence of Subpixel Corner Extraction}

The first contribution is the implementation related consistent application of subpixel accurate point measurements. We calibrated each data set with and without subpixel measurements using [2]. The column 'subpix' of Table 1 depicts the improved calibration results w.r.t to the achieved RMS. Further, Table 2 shows the effect on the standard deviation of the exterior orientation parameters and Table 3 the standard deviation of the interior orientation. Evidently using subpixel measurements provides a more accurate and stable calibration result. The RMS (decrease up to factor 3.80) as well as, e.g. the standard deviation of the exterior orientation (decrease also up to factor 3.78) decreased significantly.

\begin{tabular}{|l|c|c|c|c|c|c|}
\hline data set & standard & subpix & proposed & proposed subpix & robust subpix & mei's tool \\
\hline Fisheye1 & 0.98 & 0.84 & 0.44 & $\mathbf{0 . 2 8}$ & $\mathbf{0 . 2 8}$ & 2.85 \\
\hline Fisheye2 & 0.58 & 0.15 & 0.37 & $\mathbf{0 . 0 8}$ & $\mathbf{0 . 0 8}$ & 1.56 \\
\hline GoPro & 1.58 & 1.35 & 0.83 & $\mathbf{0 . 5 4}$ & $\mathbf{0 . 5 1}$ & 13.22 \\
\hline MiniOmni & 0.72 & 0.59 & 0.47 & $\mathbf{0 . 3 8}$ & $\mathbf{0 . 3 3}$ & 1.83 \\
\hline VMRImage & 0.60 & 0.39 & 0.39 & $\mathbf{0 . 2 5}$ & $\mathbf{0 . 2 4}$ & 0.27 \\
\hline Ladybug & 0.79 & 0.70 & 0.39 & $\mathbf{0 . 2 8}$ & $\mathbf{0 . 2 7}$ & 0.27 \\
\hline KaidanOmni & 0.64 & 0.33 & 0.45 & $\mathbf{0 . 2 2}$ & $\mathbf{0 . 2 2}$ & 1.51 \\
\hline
\end{tabular}

Table 1: RMS in pixel for all datasets. Each dataset is calibrated with six methods, i.e. standard [2], standard with subpixel corners, proposed refinement without subpixel corners, proposed refinement and robust refinement with subpixel corners and mei's toolbox [4]. Instead of the standard implementation each method uses sub-pixel point measurements.

\subsection{Influence of Joint Refinement}

The main contribution is the reformulation of the non-linear least squares problem (Eq. 11. Eq. 10. The column 'proposed' in Table 1 depicts the RMS results for all data sets. Clearly the accuracy for all data sets is significantly increased, e.g. from 0.98 to 0.44 , i.e. by factor 2.2 for the 'Fisheye1' data set.

Subsequently, we applied all changes to the calibration, i.e. one-step refinement as well as subpixel corners and a further quality increase can be observed. From the original implementation the highest decrease for the RMS (factor 7.10) 
can be observed for the 'Fisheye2' data set. Furthermore, for all data sets that contained outlier corners, i.e. where a reprojected point does not lie within the radius of 1 pixel from the extracted checkerboard corner, the robust bundle adjustment yielded even better results. From Table 1 we can derive a mean RMS decrease for each column compared to the standard implementation. The mean RMS decrease to 'subpix' is 1.27 , to 'proposed' it's 1.75 , to 'proposed subpix' it's 3.37 and to 'robust subpix' it's 3.45. This proofs the validity of each contribution. The overall performance gain for the RMS between the standard implementation [2] and our proposed method is also graphically visualized in Figure 3 .

As the RMS is only an indication of how well the estimated model describes the observations, we also assessed the quality of the estimated parameters. Since the redundancy of our proposed bundle adjustment is large, we can calculate the empirical covariance matrix $\boldsymbol{\Sigma}_{x x}\left[18\right.$. The main diagonal of $\boldsymbol{\Sigma}_{x x}$ contains the variance information of all unknowns parameters $\hat{\mathbf{U}}$. As the standard implementation separates the refinement into two steps, we extract one covariance matrix after each step separately, i.e. yielding a covariance matrix for interior and exterior orientation. Note, that the separated covariance matrices do not contain the aforementioned correlations between the calibration parameters. This statistical fact additionally reveals the disadvantage of refining interior and exterior orientation separately. Hence, a comparison of the covariance matrices between the standard and our proposed approach should only be cautiously interpreted. Table 2 depicts the median standard deviation for orientation and translation averaged over all images for all methods. The standard deviation of the exterior orientation decreased up to 5.5 times. Table 3 depicts the standard deviation for two interior orientation parameters, i.e. first affine parameter and the first polynomial coefficient. For the sake of clarity all other interior orientation parameters are omitted. The tendencies are the same.

In a last step, we studied the runtime differences of both approaches, as [1] argues that the two step refinement reduces the runtime of the calibration proce- 
dure. We assembled a script to execute all calibration related functions without user interaction and measured the execution time. The standard five step calibration procedure was carried out with only one refinement iteration. Table 4 depicts the overall results. Obviously the reduction from five to three calibration steps decreased the runtime. The elimination of the center of distortion search fully compensates for the slightly longer runtime of the non-linear refinement.

(a)

\begin{tabular}{|l|c|c|c|c|}
\hline data set & standard & subpix & proposed & robust \\
\hline & {$[\mathrm{mm}]$} & {$[\mathrm{mm}]$} & {$[\mathrm{mm}]$} & {$[\mathrm{mm}]$} \\
\hline Fisheye1 & 0.23 & 0.21 & $\mathbf{0 . 0 9}$ & $\mathbf{0 . 0 9}$ \\
\hline Fisheye2 & 1.27 & 0.34 & $\mathbf{0 . 1 9}$ & $\mathbf{0 . 1 9}$ \\
\hline GoPro & 0.58 & 0.51 & $\mathbf{0 . 3 9}$ & $\mathbf{0 . 3 8}$ \\
\hline MiniOmni & 0.56 & 0.47 & $\mathbf{0 . 2 7}$ & $\mathbf{0 . 2 3}$ \\
\hline VMRImage & 0.42 & 0.27 & $\mathbf{0 . 3 8}$ & $\mathbf{0 . 3 8}$ \\
\hline Ladybug & 0.26 & 0.22 & $\mathbf{0 . 2 7}$ & $\mathbf{0 . 2 6}$ \\
\hline KaidanOmni & 0.63 & 0.31 & $\mathbf{0 . 3 9}$ & $\mathbf{0 . 3 9}$ \\
\hline
\end{tabular}

(b)

\begin{tabular}{|c|c|c|c|}
\hline standard & subpix & proposed & robust \\
\hline$[\mathrm{mrad}]$ & {$[\mathrm{mrad}]$} & {$[\mathrm{mrad}]$} & {$[\mathrm{mrad}]$} \\
\hline 2.36 & 2.07 & $\mathbf{0 . 6 8}$ & $\mathbf{0 . 7 3}$ \\
\hline 2.39 & 0.87 & $\mathbf{0 . 4 0}$ & $\mathbf{0 . 4 0}$ \\
\hline 1.12 & 1.00 & $\mathbf{0 . 6 4}$ & $\mathbf{0 . 6 3}$ \\
\hline 4.77 & 3.93 & $\mathbf{1 . 8 3}$ & $\mathbf{1 . 6 2}$ \\
\hline 6.19 & 3.95 & $\mathbf{2 . 3 6}$ & $\mathbf{2 . 3 5}$ \\
\hline 3.23 & 3.18 & $\mathbf{1 . 6 8}$ & $\mathbf{1 . 6 3}$ \\
\hline 5.24 & 2.68 & $\mathbf{1 . 7 9}$ & $\mathbf{1 . 7 8}$ \\
\hline
\end{tabular}

Table 2: Depicts the median standard deviations $\bar{\sigma}$ for (a) position $\bar{\sigma}_{x y z}$ and (b) orientation $\bar{\sigma}_{\phi \kappa \gamma}$ for the exterior orientation of each dataset w.r.t. the calibration pattern. (a) the mean standard deviation in $x, y$ and $z$ direction of the translation vector in $[\mathrm{mm}]$. (b) the mean standard deviation of the orientation about $x, y$ and $z$ axis in [mrad]. Subpixel were used for all exepct the standard procedure.

(a)

\begin{tabular}{|l|c|c|c|c|}
\hline data set & standard & subpix & proposed & robust \\
\hline & {$[1 \mathrm{e}-3]$} & {$[1 \mathrm{e}-3]$} & {$[1 \mathrm{e}-3]$} & {$[1 \mathrm{e}-3]$} \\
\hline Fisheye1 & 0.41 & 0.35 & $\mathbf{0 . 1 3}$ & $\mathbf{0 . 2 3}$ \\
\hline Fisheye2 & 0.49 & 0.13 & $\mathbf{0 . 0 8}$ & $\mathbf{0 . 0 8}$ \\
\hline GoPro & 0.24 & 0.20 & $\mathbf{0 . 1 8}$ & $\mathbf{0 . 1 8}$ \\
\hline MiniOmni & 0.41 & 0.33 & $\mathbf{0 . 2 6}$ & $\mathbf{0 . 2 3}$ \\
\hline VMRImage & 0.39 & 0.24 & $\mathbf{0 . 4 5}$ & $\mathbf{0 . 4 5}$ \\
\hline Ladybug & 0.72 & 0.60 & $\mathbf{0 . 3 0}$ & $\mathbf{0 . 2 9}$ \\
\hline KaidanOmni & 1.72 & 0.83 & $\mathbf{0 . 7 2}$ & $\mathbf{0 . 7 2}$ \\
\hline
\end{tabular}

Table 3: Depicts the median standard deviations $\bar{\sigma}$ for (a) the first affine parameter $\bar{\sigma}_{c}$ and (b) the polynom coefficient $\bar{\sigma}_{a_{0}}$. Subpixel were used for all except the standard procedure.

(b)

\begin{tabular}{|c|c|c|c|}
\hline standard & subpix & proposed & robust \\
\hline$[1 \mathrm{e}-3]$ & {$[1 \mathrm{e}-3]$} & {$[1 \mathrm{e}-3]$} & {$[1 \mathrm{e}-3]$} \\
\hline 2.02 & 1.71 & $\mathbf{0 . 6 9}$ & $\mathbf{0 . 6 9}$ \\
\hline 2.06 & 0.51 & $\mathbf{0 . 3 2}$ & $\mathbf{0 . 3 2}$ \\
\hline 0.92 & 0.79 & $\mathbf{0 . 5 3}$ & $\mathbf{0 . 5 2}$ \\
\hline 7.30 & 6.13 & $\mathbf{3 . 6 6}$ & $\mathbf{3 . 2 7}$ \\
\hline 5.28 & 3.21 & $\mathbf{2 . 3 1}$ & $\mathbf{2 . 3 1}$ \\
\hline 2.65 & 2.40 & $\mathbf{1 . 5 3}$ & $\mathbf{1 . 4 8}$ \\
\hline 18.47 & 8.59 & $\mathbf{5 . 2 4}$ & $\mathbf{5 . 2 2}$ \\
\hline
\end{tabular}




\begin{tabular}{|c|c|c|c|c|c|c|c|c|}
\hline data set: & & Fisheye1 & Fisheye2 & GoPro & MiniOmni & VMRImage & Ladybug & KaidanOmni \\
\hline standard & {$[\mathrm{s}]$} & 48 & 68 & 211 & 77 & 35 & 28 & 32 \\
\hline proposed & {$[\mathrm{s}]$} & $\mathbf{3 7}$ & $\mathbf{4 3}$ & $\mathbf{1 9 6}$ & $\mathbf{5 8}$ & $\mathbf{1 8}$ & $\mathbf{1 2}$ & $\mathbf{1 8}$ \\
\hline
\end{tabular}

Table 4: Runtime difference between the standard procedure and our proposed 3-step procedure.

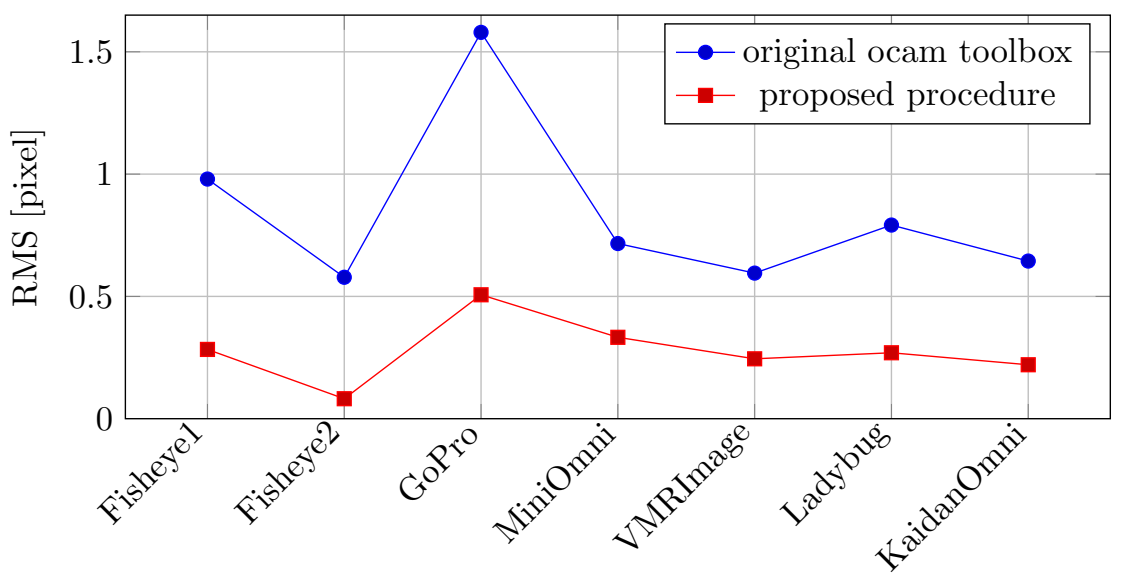

Figure 3: Overall performance gain for the RMSE between the standard implementation [2] and our proposed non-linear refinement with subpixels.

\subsection{Comparison to Mei's toolbox}

Finally, we compare the improved calibration procedure to the method proposed in [3] whose Matlab implementation is also available online (4]). The last column of Table 1 depicts the results. Clearly, only 3 out of 8 cameras could be accurately calibrated. For 'VMRImage' and 'Ladybug', a similar accuracy w.r.t the RMS is achievied. The remaining 5 cameras could not be accurately calibrated. To automatically extract all checkerboard corners [3] first estimates a planar homography from 4 manually selected corner points. Then, the remaining checkerboard points are projected to their estimated image location and a local subpixel corner search is performed. The estimation of the planar homography is based on initial values such as the generalized focal length and the center of distortion (see [3] for details). Latter is estimated from the mirror border (in the omnidirectional case) or initialized as the image center. Since 
some of the cameras are low-cost and the center of distortion can have a large offset to the image center, the initial estimates are significantly biased and the calibration procedure can obviously not recover from that. This is confirmed by the fact, that we were not able to accurately calibrate our fisheye cameras 'Fisheye1', 'Fisheye2'. For both cameras the center of distortion deviates more than 10 pixels from the image center. After performing several calibrations on all data sets, we found that a large percentage of points are falsely extracted. For some data sets, we improved the calibration result by manual tuning of the search window of the subpixel corner search or by using better initial estimates, e.g. for the center of distortion but this is not desirable in the sense of a fully automatic calibration routine. Note, that our proposed calibration procedure does not depend on any manual interaction.

\section{Conclusions}

In this paper, we extended the widely used method and implementation for fisheye and omnidirectional camera calibration of [1, 2, 5]. Apart from using subpixel accurate corner measurements we reformulated the non-linear least squares refinement and applied a residual function that allows to jointly optimize all calibration parameters. We further reduced the necessary calibration steps to three and evaluated the results with various data sets including wide-angle, fisheye and omnidirectional imaging systems. It showed that a significant performance increase (RMS up to factor 7.1) could be achieved. Since the automatic corner extraction yields false locations from time to time, we extended the non-linear refinement with an M-estimator to make it robust against outliers. Finally, Figure 4 depicts a visual comparison of the reprojected corners between the standard method on the left side and our proposed method on the right. Evidently, after a calibration with the proposed method, the reprojected points (magenta circles) coincide much better with the extracted corner points (red crosses). A Matlab implementation of all contributions is available online (see Section 1) and can be used as an addon to the existing toolbox [2]. 


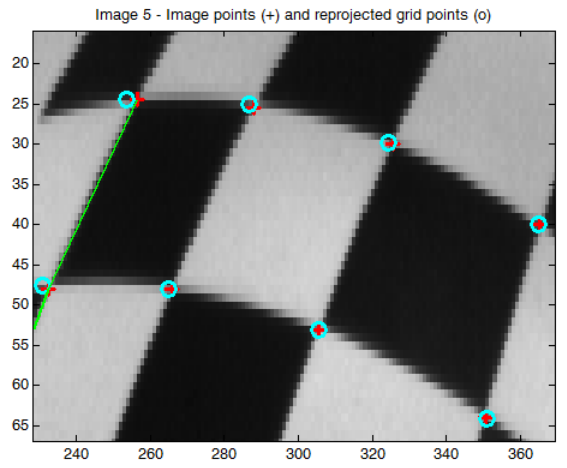

(a) standard method

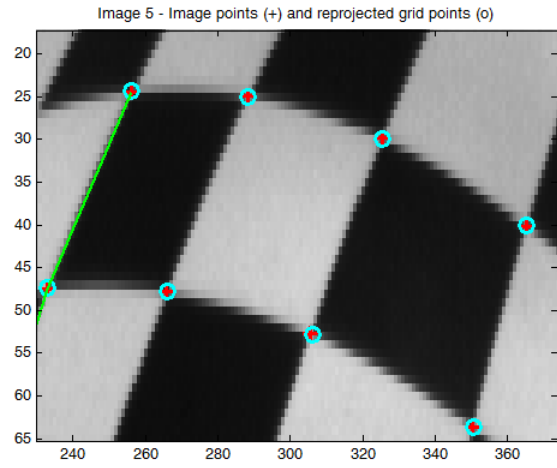

(b) proposed method

Figure 4: Visual comparison of the reprojected (magenta circles) corner points (red crosses) for (a) the standard method and (b) our proposed robust refinement. This is an arbitrary region of the checkerboard from image 5 of the 'Fisheye2' data set.

Acknowledgments. This project was partially funded by the DFG research group FG 1546 "Computer-Aided Collaborative Subway Track Planning in Multi-Scale 3D City and Building Models".

\section{References}

[1] D. Scaramuzza, A. Martinelli, R. Siegwart, A flexible technique for accurate omnidirectional camera calibration and structure from motion, in: Computer Vision Systems, 2006 ICVS'06. IEEE International Conference on, IEEE, 2006, pp. 45-45.

[2] Davide Scaramuzza, Ocamcalib: Omnidirectional camera calibration toolbox for matlab, https://sites.google.com/site/scarabotix/ ocamcalib-toolbox, visited in february 2014 (2014).

[3] C. Mei, P. Rives, Single view point omnidirectional camera calibration from planar grids, in: IEEE International Conference on Robotics and Automation, 2007.

[4] C. Mei, Omnidirectional calibration toolbox, http://www.robots.ox.ac. uk/ cmei/Toolbox.html, visited in february 2014 (2014). 
[5] D. Scaramuzza, A. Martinelli, R. Siegwart, A toolbox for easily calibrating omnidirectional cameras, in: Intelligent Robots and Systems, 2006 IEEE/RSJ International Conference on, IEEE, 2006, pp. 5695-5701.

[6] B. Micušlk, Two-view geometry of omnidirectional cameras, Ph.D. thesis, Czech Technical University (2004).

[7] J. Kannala, S. S. Brandt, A generic camera model and calibration method for conventional, wide-angle, and fish-eye lenses, Pattern Analysis and Machine Intelligence, IEEE Transactions on 28 (8) (2006) 1335-1340.

[8] C. Geyer, K. Daniilidis, A unifying theory for central panoramic systems and practical implications, in: Computer VisionECCV 2000, Springer, 2000, pp. $445-461$.

[9] L. Puig, J. Bermúdez, P. Sturm, J. Guerrero, Calibration of omnidirectional cameras in practice: A comparison of methods, Computer Vision and Image Understanding 116 (1) (2012) 120 - 137. doi:http://dx.doi.org/10.1016/j.cviu.2011.08.003.

URL http://www.sciencedirect.com/science/article/pii/ S1077314211001858

[10] Z. Zhang, Flexible camera calibration by viewing a plane from unknown orientations, in: Computer Vision, 1999. The Proceedings of the Seventh IEEE International Conference on, Vol. 1, IEEE, 1999, pp. 666-673.

[11] B. Micušlk, T. Pajdla, Structure from motion with wide circular field of view cameras, Pattern Analysis and Machine Intelligence, IEEE Transactions on 28 (7) (2006) 1135-1149.

[12] O. D. Faugeras, Q.-T. Luong, S. J. Maybank, Camera self-calibration: Theory and experiments, in: Computer VisionECCV'92, Springer, 1992, pp. $321-334$. 
[13] D. Schneider, E. Schwalbe, H.-G. Maas, Validation of geometric models for fisheye lenses, ISPRS Journal of Photogrammetry and Remote Sensing 64 (3) (2009) 259-266.

[14] L. Puig, Y. Bastanlar, P. Sturm, J. J. Guerrero, J. Barreto, Calibration of central catadioptric cameras using a dlt-like approach, International Journal of Computer Vision 93 (1) (2011) 101-114.

[15] M. Rufli, D. Scaramuzza, R. Siegwart, Automatic detection of checkerboards on blurred and distorted images, in: Intelligent Robots and Systems, 2008. IROS 2008. IEEE/RSJ International Conference on, IEEE, 2008, pp. $3121-3126$.

[16] O. Frank, R. Katz, C.-L. Tisse, H. F. Durrant-Whyte, Camera calibration for miniature, low-cost, wide-angle imaging systems., in: BMVC, Citeseer, 2007, pp. 1-10.

[17] J. Bouguet, Camera calibration toolbox for matlab http: //www.vision.caltech.edu/bouguetj/calib_doc/index.html, visited february 2014 [cited 26.02.2014].

URL http://www.vision.caltech.edu/bouguetj/calib_doc/index. html

[18] C. D. Ghilani, Adjustment computations: spatial data analysis, John Wiley \& Sons, 2010. 